\title{
Multilevel Space Vector PWM Control Schemes for a Flying- Capacitor Inverter
}

\author{
S. J. Watkins and L Zhang \\ School of Electronic and Electrical Engineering, The University of Leeds, Leeds, LS2 9JT \\ E-mail: 1.zhang@ee.leeds.ac.uk Tel: 01132332020 Fax: 01133432032
}

Keywords: Multilevel Inverter, Space Vector Modulation

\begin{abstract}
The paper presents three different computational schemes for achieving space vector pulse width modulation, in the control of a multilevel flying capacitor inverter. Each scheme uses the same method of achieving cell-capacitor voltage balancing. Results of realistic simulations are used to compare and contrast these approaches. The timing control of the inverter switches and output performance of each method are presented and the most suitable one is highlighted.
\end{abstract}

\section{Introduction}

Multilevel power converters are gaining increasing interest for medium voltage systems in static power conversion and variable speed drives due to their operational advantages. Suitable fast switching high-voltage, high-current IGBTs are now available for practical implementations and R\&D emphasis is on optimising the control to achieve low waveform distortion and high efficiency. There are several control methods for achieving low harmonic content in the sinusoidal output voltage. The simplest form utilises the voltage levels in forming a staircase shaped waveform approximating a sine-wave. Other techniques apply conventional PWM scheme whereby the inverter switching is controlled from signals generated by comparing a sinusoidal reference to a set of triangular carriers. The control scheme now gaining most interest is space vector PWM (SV-PWM) [1] where the three-phase stationary reference frame voltages for each inverter switching states are mapped to the complex two-phase orthogonal $\alpha-\beta$ plane. The reference voltage is represented as a vector in the plane and duty-cycles are computed for the selected switching state vectors in proximity to the reference. In multilevel inverters, the control complexity increases with the voltage level capability, making it more difficult to select the appropriate vectors.

This paper presents the results of an investigation into SVPWM control techniques and their application to a multilevel flying-capacitor inverter [2,3]. There have been a number of SV-PWM computational algorithms presented. They address the problem in achieving real-time control of the inverter power switches, while attaining high output power quality with a view to implementation in commercially available hardware such as a DSP. The most popular schemes split the hexagonal boundary of the space vectors in the complex plane into sector or triangular regions bounded $[4,5,6]$. Computation is commonly done through the look-up table approach.

The work presented in this paper adopts a systematic approach to identify the most suitable SV-PWM scheme applicable to the control of the flying-capacitor multilevel inverter [7]. Three different methods are investigated; including the one using only the two-level eight-vector approach to the one considers all vectors in a multilevel inverter. The rationale in selecting vectors and subsequent procedures for duty cycle computations for each of them are discussed. Their performance in terms of the output voltage harmonic quality and computational efficiency are compared. The control scheme must incorporate a cell-capacitor voltage balancing method by ensuring that cycling of the different switching states for each voltage level takes place. Such a method is described and presented in the paper. The variations in output harmonic distortion on an inverter with realistic power components under different SV-PWM schemes with voltage balancing control are studied and results are presented.

\section{Space Vector Representation}

The spread of space vectors for an N-cell three-phase flyingcapacitor inverter always lie within a bounded hexagonal region. The vectors forming the boundary are associated with one or two phases with full-voltage switching states. Symmetry within the hexagon allows a systematic vector selection approach to be developed firstly in one $\pi / 3$ triangular region and applied through the complete $2 \pi$ cycle.

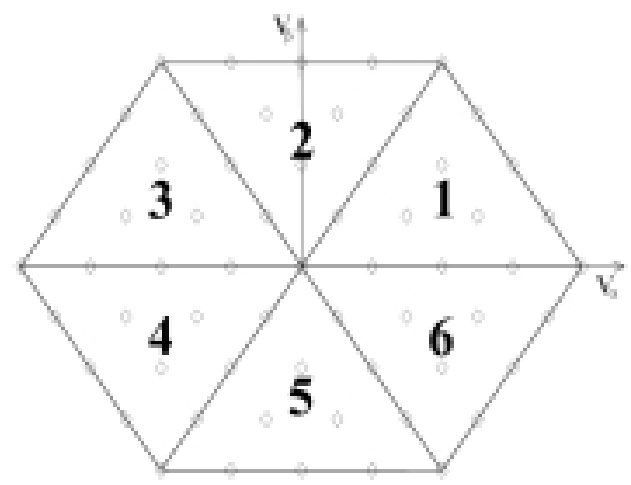

Figure 1: Space-Vector Sector Representation 
Figure 1 shows the main primary sector triangular regions in the $\alpha-\beta$ plane for a 4 -cell inverter. The individual points within the hexagon represent the individual vectors for different switching states when the cell-capacitor voltages are at the optimum balanced levels.

\subsection{SV-PWM Method \#1}

The basic space vector modulation scheme has 8 voltage vectors representing all possible conditions when any phase is supplying either zero volts or $\mathrm{V}_{\mathrm{dc}}$. In a multilevel inverter there are additional voltage vectors for the intermediary voltage conditions. For a simplified space vector scheme, the intermediary voltage vectors can be ignored and only the voltage vectors from the centre to the hexagon vertices used. Figure 1 illustrates the space vector diagram for a 4-cell inverter where all upper switches conducting in a phase are represented by a 4 . This simplified scheme can utilise carrierbased PWM to produce the firing signals since the computed timing for the vectors in each operating triangle can be used to form the reference signal for each phase.

For the sector triangle bounded by vectors $\vec{V}_{1}$ and $\vec{V}_{2}$ where $\phi$ $<\pi / 3$ the standard equations for calculating the time width of a vector are as follows

$$
T_{1}=T_{s} M\left(\cos \theta-\frac{\sin \theta}{\sqrt{3}}\right), \quad T_{2}=\frac{2}{\sqrt{3}} T_{s} M \sin \theta
$$$$
T_{0}=T_{s}-T_{1}-T_{2}
$$
and

subsequently the magnitudes of vectors approximating $\hat{V}_{\text {ref }}$ as shown in Figure 1 are given as below

$$
\begin{aligned}
& \hat{V}_{1}=\hat{V}_{r e f}\left(\cos \phi-\frac{\sin \phi}{\sqrt{3}}\right) \\
& \hat{V}_{2}=\frac{2 \hat{V}_{r e f} \sin \phi}{\sqrt{3}} \\
& \hat{V}_{0}=\frac{\sqrt{3}}{2} V_{D C}-\hat{V}_{1}-\hat{V}_{2}
\end{aligned}
$$

where M, the modulation index, equals $\hat{V}_{r e f} / \hat{V}_{D C}$. Inspecting the timing diagram for space vector modulation shown in Figure 2, the average phase voltages can be given as

$V_{a}=\frac{1}{T_{S}} \frac{2}{\sqrt{3}} V_{D C}\left(\frac{T_{0}}{2}+T_{1}+T_{2}\right), \quad V_{b}=\frac{1}{T_{S}} \frac{2}{\sqrt{3}} V_{D C}\left(\frac{T_{0}}{2}+T_{2}\right)$ and $V_{c}=\frac{1}{T_{S}} \frac{T_{0}}{\sqrt{3}} V_{D C}$

These may be expressed using the magnitudes of voltage vectors given in Equations (1) - (3), thus we have
$V_{a}=\frac{2}{\sqrt{3}}\left(\frac{\hat{V}_{0}}{2}+\hat{V}_{1}+\hat{V}_{2}\right), V_{b}=\frac{2}{\sqrt{3}}\left(\frac{\hat{V}_{0}}{2}+\hat{V}_{2}\right)$ and $V_{c}=\frac{\hat{V}_{0}}{\sqrt{3}}$

Separate sets of reference equations can be produced for each sector triangle by decomposing the individual phase voltage states from each space vector. These are shown in Table 1 for phase A. Phase B and C reference voltages are the same as phase but offset by $2 \pi / 3$ and $4 \pi / 3$ respectively.

\begin{tabular}{|c|c|}
\hline Phase Angle & $\boldsymbol{V}_{\boldsymbol{a}}$ \\
\hline $0 \leq \phi<\pi / 3$ & $\frac{2}{\sqrt{3}}\left(\frac{\hat{V}_{0}}{2}+\hat{V}_{1}+\hat{V}_{2}\right)$ \\
\hline$\pi / 3 \leq \phi<2 \pi / 3$ & $\frac{2}{\sqrt{3}}\left(\frac{\hat{V}_{0}}{2}+\hat{V}_{1}\right)$ \\
\hline $2 \pi / 3 \leq \phi<\pi$ & $\frac{\hat{V}_{0}}{\sqrt{3}}$ \\
\hline$\pi \leq \phi<4 \pi / 3$ & $\frac{\hat{V}_{0}}{\sqrt{3}}$ \\
\hline $4 \pi / 3 \leq \phi<5 \pi / 3$ & $\frac{2}{\sqrt{3}}\left(\frac{\hat{V}_{0}}{2}+\hat{V}_{2}\right)$ \\
\hline $5 \pi / 3 \leq \phi<2 \pi$ & $\frac{2}{\sqrt{3}}\left(\frac{\hat{V}_{0}}{2}+\hat{V}_{1}+\hat{V}_{2}\right)$ \\
\hline
\end{tabular}

Table 1: Simplified Space Vector Modulation Phase A Reference Voltage Equations

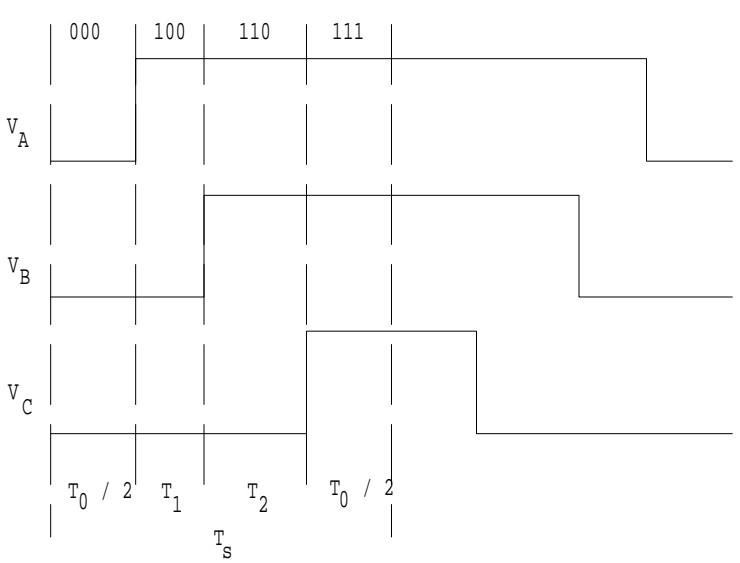

Figure 2: Space Vector Modulation Timing Diagram

The net result is a reference signal centred on $V_{d c} / 2$ with the waveform shown in figure 3 . This waveform is a composite of a cosine wave plus a third order triangular wave. The basic form of reference equation for phase A can be approximated to:

$$
V_{r e f}=V_{D C}+\frac{3 V}{4} \cos \phi \pm \frac{3 V}{8}\left(\frac{\pi}{6}-\left(\phi-t \frac{\pi}{3}\right)\right)
$$




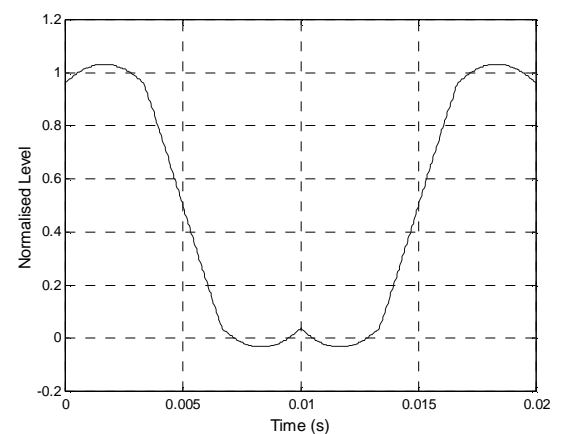

Figure 3: Phase Voltage Reference

\subsection{SV-PWM Method \#2}

The firing signal design philosophy can be further extended to incorporate additional voltage vectors in the calculation of the phase voltage reference by including the intermediary vectors between the primary ones used in the previous section. These vectors are shown in the first triangular sector of the space vector diagram of Figure 4 . The voltage magnitude calculations for the first angular region $(0 \leq \phi<\pi / 6)$ in the sector are as follows:

$$
\begin{aligned}
& \hat{V}_{1}=\hat{V}_{r e f}(\cos \phi-\sqrt{3} \sin \phi) \\
& \hat{V}_{2}=2 \hat{V}_{r e f} \sin \phi \\
& \hat{V}_{0}=\frac{\sqrt{3}}{2} V_{D C}-\hat{V}_{1}-\hat{V}_{2}
\end{aligned}
$$

The phase reference voltages are computed in the same manner as before. This time when the vector state is 2 , the summation uses only half the voltage length of the vector.

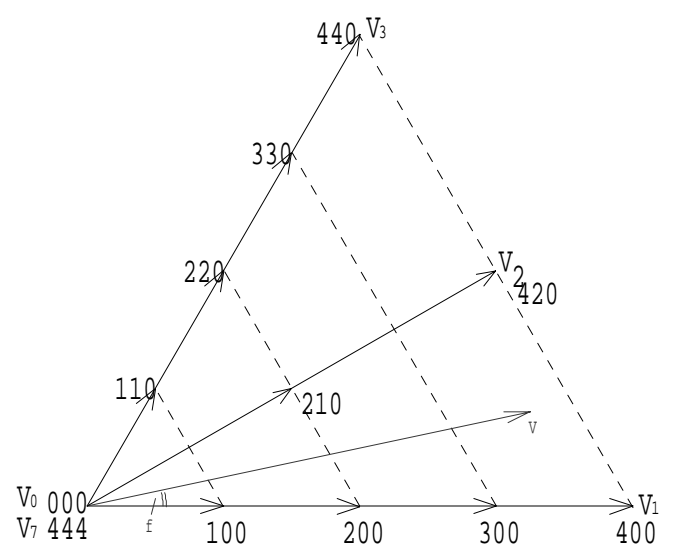

Figure 4: First Triangular Sector Phase Diagram

The phase reference voltages for phase A for all angles are shown in the Table 2. The pattern is the same for phases $B$ and $C$, offset by $2 \pi / 3$ and $4 \pi / 3$ respectively.

\begin{tabular}{|c|c|}
\hline Phase Angle & $V_{a}$ \\
\hline $0 \leq \phi<\pi / 3$ & $\frac{2}{\sqrt{3}}\left(\frac{\hat{V}_{0}}{2}+\hat{V}_{1}+\hat{V}_{2}\right)$ \\
\hline$\pi / 3 \leq \phi<\pi / 2$ & $\frac{2}{\sqrt{3}}\left(\frac{\hat{V}_{0}}{2}+\hat{V}_{1}+\frac{\hat{V}_{2}}{2}\right)$ \\
\hline$\pi / 2 \leq \phi<2 \pi / 3$ & $\frac{1}{\sqrt{3}}\left(\hat{V}_{0}+\hat{V}_{1}\right)$ \\
\hline $2 \pi / 3 \leq \phi<4 \pi / 3$ & $\frac{1}{\sqrt{3}} \hat{V}_{0}$ \\
\hline $4 \pi / 3 \leq \phi<3 \pi / 2$ & $\frac{1}{\sqrt{3}}\left(\hat{V}_{0}+\hat{V}_{2}\right)$ \\
\hline $3 \pi / 2 \leq \phi<5 \pi / 3$ & $\frac{2}{\sqrt{3}}\left(\frac{\hat{V}_{0}}{2}+\frac{\hat{V}_{1}}{2}+\hat{V}_{2}\right)$ \\
\hline $5 \pi / 3 \leq \phi<2 \pi$ & $\frac{2}{\sqrt{3}}\left(\frac{\hat{V}_{0}}{2}+\hat{V}_{1}+\hat{V}_{2}\right)$ \\
\hline
\end{tabular}

Table 2: Reference Voltage Equations for Phase A

The reference signal, centred on $\mathrm{V}_{\mathrm{dc}} / 2$, and has the form shown in Figure 5. The waveform is again a composite of a cosine wave plus a triangular wave.

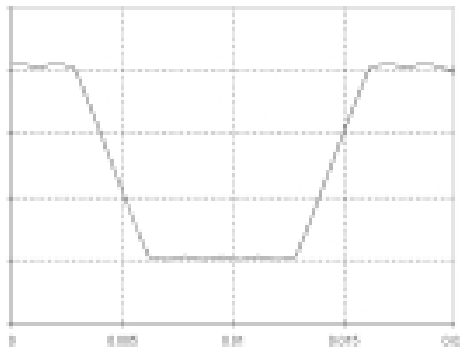

Figure 5: Extended Hybrid Space Vector Phase Reference Signal

\subsection{SV-PWM Method \#3}

The method is described with reference to sector 1 . In this sector the base is defined as the line joining switching states $[0,0,0]$ and $[4,0,0]$, which represents increasing voltage level in phase A. Level lines are defined in this sector along increasing voltage levels in phase B. Figure 6 illustrates the switching states in sector 1 and their relationship to each other.

This algorithm first identifies the level line where the first corner state space vector lies on. Mathematically, level $L$ is found from the normalised reference amplitude $V_{\text {ref }}$ which satisfies the following equation:

$(L+1) /\left(\cos \theta+\tan \frac{\pi}{6} \cdot \sin \theta\right)>\hat{V}_{r e f} \geq L /\left(\cos \theta+\tan \frac{\pi}{6} \cdot \sin \theta\right)$

The normalised amplitude is found by multiplying the ratio of actual reference vector amplitude to $V_{D C}$ by 1.5 . Figure 7 
shows graphically an example where $\mathrm{L}$ is equal to 3 for the given reference vector with normalized amplitude being 2.87 .

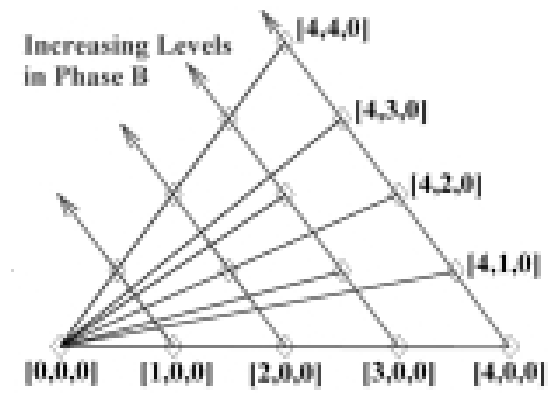

Figure 6: Sector 1 Vector Trajectories Using Phase A and B Only

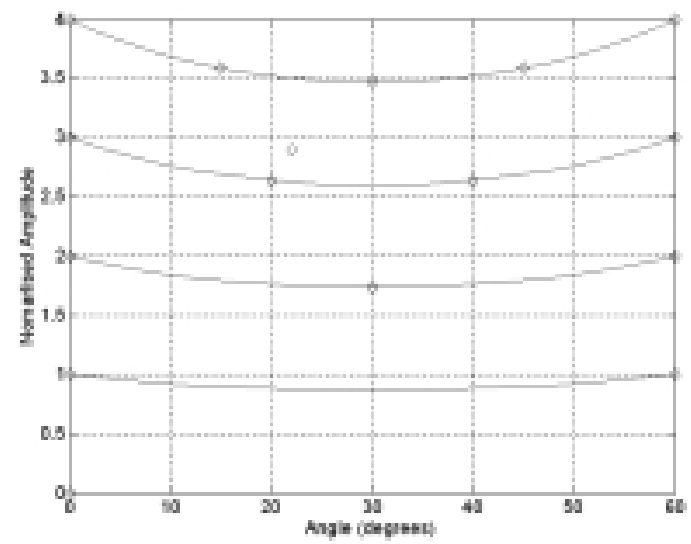

Figure 7: Sector 1 Level Section

The next step is to progress from the base level vector by incrementing the phase B level until the starting space vector is found. The mathematical criterion for this uses the reference vector phase $\theta$ and $B$ satisfies the following equation:

$$
\arctan \left(\frac{0.5(B+1)}{(L-0.5(B+1))}\right)>\theta \geq \arctan \left(\frac{0.5 B}{(L-0.5 B)}\right)
$$

Therefore, in sector 1 , the starting space vector $\hat{V}_{s}$ is [L B 0]. The first space vector is found by progressing the starting vector in the negative $\mathrm{C}$ direction. Since $\mathrm{C}$ is already zero, then incrementing both $\mathrm{A}$ and $\mathrm{B}$ will achieve the same result. Therefore, the first vector $\hat{V}_{0}$ is $[(\mathrm{L}+1)(\mathrm{B}+1) 0]$. This vector sits on three possible triangular regions in which the reference vector lies. The selection of the other two space vectors can be found from analysing the phase of the vector joining the first vector from the reference.

If the phase of the vector $\hat{V}_{r e f}-\hat{V}_{0}$ is greater than the phase of vector $\hat{V}_{s}-\hat{V}_{0}$, then the starting vector $\hat{V}_{s}$ is the second of the vector triplet and the third vector is found by decrementing $\mathrm{V} 0$ in phase $\mathrm{B}$, thus giving the set of vectors [L B 0], $[(\mathrm{L}+1)$ $(\mathrm{B}+1) 0],[(\mathrm{L}+1) \mathrm{B} 0]$.

If the phase of the vector $\hat{V}_{r e f}-\hat{V}_{0}$ is less than the phase of vector $\hat{V}_{s}-\hat{V}_{0}$ and less than $\pi$, then the starting vector $\hat{V}_{s}$ is the second of the vector triplet and the third vector is found by decrementing $\hat{V}_{0}$ in phase $\mathrm{A}$, thus giving the set of vectors [L B 0], [(L+1) $(\mathrm{B}+1) 0]$, [L (B +1) 0].

If the phase of the vector $\hat{V}_{r e f}-\hat{V}_{0}$ does not meet the above criteria, then the second vector is found by decrementing $\hat{V}_{0}$ in phase $\mathrm{A}$, and the third vector is found by incrementing $\hat{V}_{0}$ in phase $B$. Thus giving the set of vectors $[(L(B+1) 0]$, $[(\mathrm{L}+1)(\mathrm{B}+1) 0],[\mathrm{L}(\mathrm{B}+2) 0]$.

To illustrate the identification of the vector triplet, Figure 8 shows the progression through the space vectors to find the triplet for the three possible results.

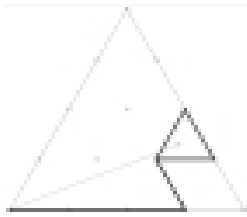

(a): Triplet 1

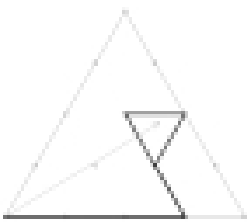

(b): Triplet 2

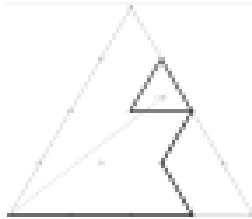

(c): Triplet 3
Figure 8: Vector Triplet Selection

The above described algorithm for identifying the vector triplet to be used in the space vector PWM scheme can easily be coded in software. The procedure in the other space vector sectors follows the same procedure by using symmetry. Table 3 lists the different parameters used in each sector.

Once the three switching state vectors are found which form a triangle in the $\alpha-\beta$ plane around the reference vector, then the PWM duty cycle terms can be calculated. Figure 9 shows the vector diagram for this modulation method.

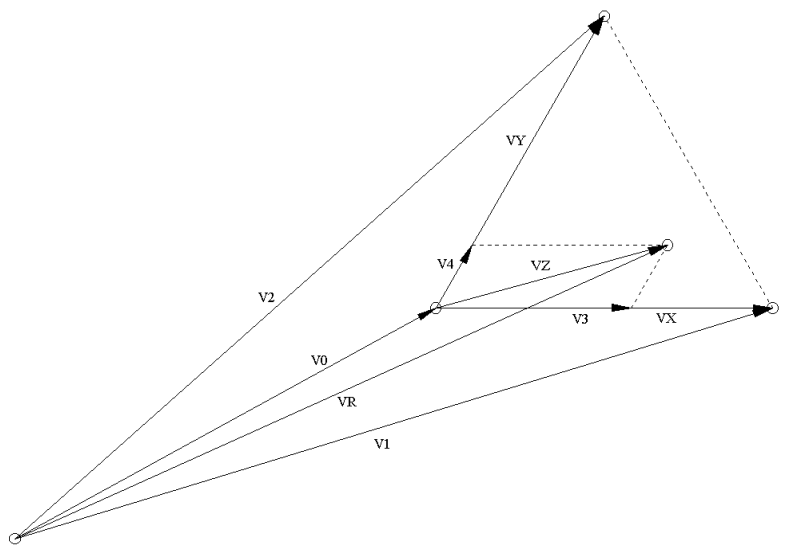

Figure 9: Space Vector Diagram 


\begin{tabular}{|c|c|c|c|c|c|c|c|c|c|c|}
\hline \multirow{2}{*}{ 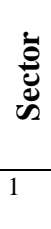 } & \multirow{2}{*}{ 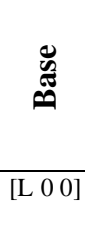 } & \multirow{2}{*}{$\begin{array}{l}\frac{\vec{E}}{\bar{D}} \\
+\mathrm{B}\end{array}$} & \multirow{2}{*}{ 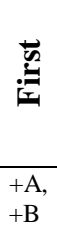 } & \multirow{2}{*}{$\frac{0}{80}$} & \multicolumn{2}{|c|}{ 离 } & \multicolumn{2}{|c|}{ 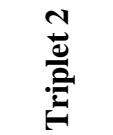 } & \multicolumn{2}{|c|}{$\frac{m}{0}$} \\
\hline & & & & & $-B$ & $+\mathrm{B}$ & $-A$ & $+\mathrm{B}$ & $-\mathrm{A}$ & $+\mathrm{B}$ \\
\hline 2 & [4 L 0] & $-A$ & $+\mathrm{B}$ & $4 \pi / 3$ & $+\mathrm{A}$ & $-A$ & $+\mathrm{C}$ & $-A$ & $+\mathrm{C}$ & $-\mathrm{A}$ \\
\hline 3 & {$\left[\begin{array}{lll}0 & \mathrm{~L} & 0\end{array}\right]$} & $+\mathrm{C}$ & $\begin{array}{l}+\mathrm{B}, \\
+\mathrm{C}\end{array}$ & $5 \pi / 3$ & $-\mathrm{C}$ & $+\mathrm{C}$ & $-B$ & $+\mathrm{C}$ & $-B$ & $+\mathrm{C}$ \\
\hline 4 & {$\left[\begin{array}{lll}0 & 4 & \mathrm{~L}\end{array}\right]$} & $-B$ & $+\mathrm{C}$ & 0 & $+\mathrm{B}$ & $-B$ & $+\mathrm{A}$ & $-B$ & $+\mathrm{A}$ & $-B$ \\
\hline 5 & {$\left[\begin{array}{lll}0 & 0 & \mathrm{~L}\end{array}\right]$} & $+\mathrm{A}$ & $\begin{array}{l}+\mathrm{A}, \\
+\mathrm{C}\end{array}$ & $\pi / 3$ & $-A$ & $+\mathrm{A}$ & $-\mathrm{C}$ & $+\mathrm{A}$ & $-\mathrm{C}$ & $+\mathrm{A}$ \\
\hline 6 & {$\left[\begin{array}{lll}L & 0 & 4\end{array}\right]$} & $-\mathrm{C}$ & $+\mathrm{A}$ & $2 \pi / 3$ & $+\mathrm{C}$ & $-\mathrm{C}$ & $+\mathrm{B}$ & $-\mathrm{C}$ & $+B$ & $-\mathrm{C}$ \\
\hline
\end{tabular}

Table 3: Algorithm Sector Parameters

The vector equation to be solved to obtain the PWM duty cycle terms is:

$$
\hat{V}_{z}=D_{x} \hat{V}_{x}+D_{y} \hat{V}_{y}
$$

where

$$
\begin{aligned}
& \hat{V}_{x}=\hat{V}_{1}-\hat{V}_{0} \\
& \hat{V}_{y}=\hat{V}_{2}-\hat{V}_{0} \\
& \hat{V}_{z}=\hat{V}_{r}-\hat{V}_{0}
\end{aligned}
$$

Two simultaneous equations are obtained from the $\alpha$ and $\beta$ terms which can then be solved.

$$
\begin{aligned}
& a_{z}=D_{x} a_{x}+D_{y} a_{y} \\
& \beta_{z}=D_{x} \beta_{x}+D_{y} \beta_{y}
\end{aligned}
$$

The complete PWM switching pattern is the combined phase $\mathrm{A}, \mathrm{B}$ and $\mathrm{C}$ switching states of vector $\hat{V}_{0}$ plus the pulse width modulated parts of $\hat{V}_{x}$ and $\hat{V}_{y}$. By convention, the pattern is symmetrical around the mid-point in the PWM period.

\subsection{SV-PWM Voltage Balancing Circuit}

The intermediary voltage states in the flying-capacitor inverter can be achieved by more than one current path through the inverter by energising a different combination of the power switches. The current flow can then involve charging or discharging of the cell-capacitors. To maintain balanced operation, the mean charge in all cell-capacitors must remain zero in the steady-state. This will also ensure that the power dissipation in each power switch is the same providing thermal balance. If the sum of the conducting periods over several inverter cycles for each intermediary switching state is equal, then balanced operation is achieved. One method for achieving this is to use a BALANCING CLOCK which has a period which is a prime number multiple of four operating cycles in the four level inverter case [8]. The logic block diagram is shown in Figure 10. This is used to multiplex ie swap around the switching states, so that from one operating cycle to the next different intermediary switching states are used. The simulation of the inverter operation shows that this scheme helps balance the cellcapacitor voltages regardless of PWM switching frequency and operating cycle period.

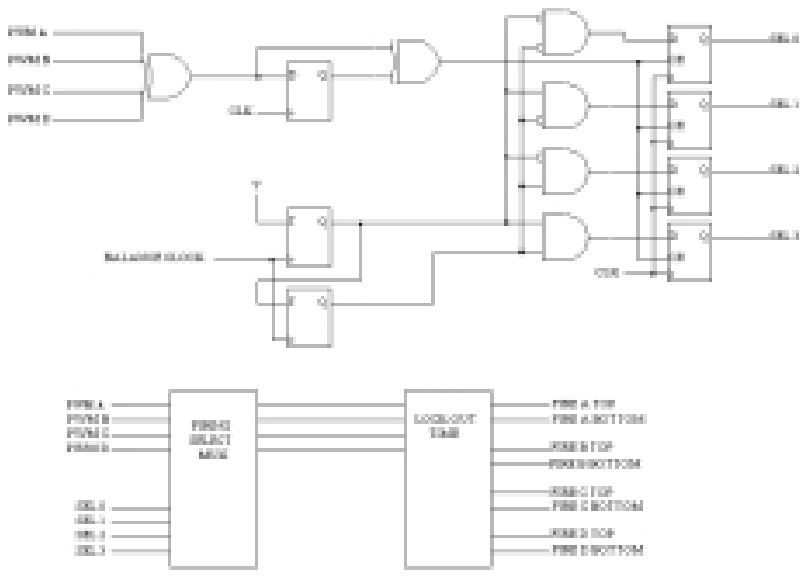

Figure 10: Inverter balancing logic

\section{Simulation Results}

Simulations of a four-cell, three-phase flying-capacitor circuit with an individual unit cell-capacitance of $47 \mathrm{uF}$ have been run for each method described. The DC-link voltage is 4000 $\mathrm{V}$ and the frequency of the synthesised output voltage is 50 $\mathrm{Hz}$ with an equivalent $m_{a}=0.9$. The simulation includes the losses in the power switches and voltage variation in the cellcapacitors so there is a reduction in the actual output voltage compared to the desired ideal level. The PWM switching frequency is $750 \mathrm{~Hz}\left(m_{f}=15\right)$. The inverter output waveforms and frequency spectrum for the three methods are shown in Figures $11-16$.

Superficially, there is very little difference between each modulation scheme. Using the total harmonic distortion (THD) percentage of the fundamental as a quality mark, method \#3 has the lowest THD $=21.6 \%$, compared to $21.9 \%$ for method \#1 and $22.2 \%$ for method \#2. These results suggestion that the computationally more intensive method using the three nearest vectors in the multilevel inverter has no significant benefit compared to using the basic SV-PWM computation applied with multilevel carriers. 


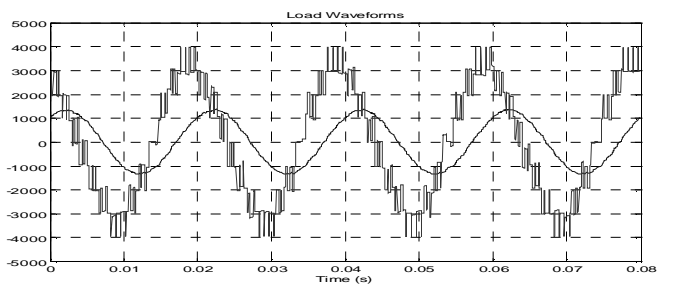

Figure 11: Method \#1 Line-to-Line Voltage and Phase Current

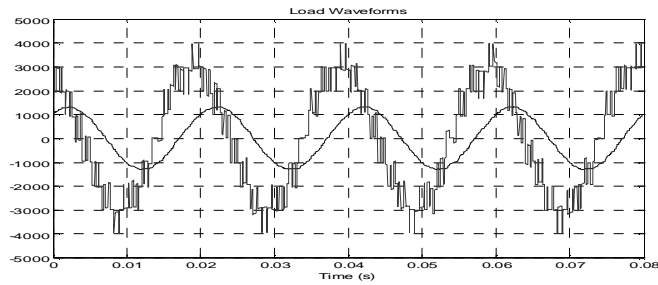

Figure 13: Method \#2 Line-to-Line Voltage and Phase Current

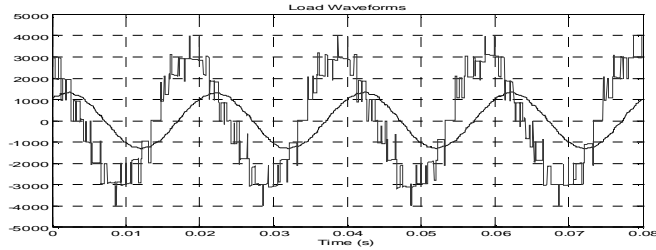

Figure 15: Method \#3 Line-to-Line Voltage and Phase Current

\section{Conclusions}

The main aim of this work is to develop computational methods for implementing SV-PWM on a multilevel inverter. The simulation results suggest that good output power quality can be achieved by using the basic space vector computation method and then transposing the timings into a reference which can be used with a set of triangular carriers, one for each voltage level. The computation is done assuming ideal cell-capacitor voltages and so further work is required to investigate whether the method \#3 computation using the nearest three vectors has any benefit when applied to the actual voltage vectors in real-time.

\section{References}

1. Van der Broeck, H.W., Skudelny, H.-C. and Stanke, G.V., "Analysis and realization of a pulsewidth modulator based on voltage space vectors", IEEE Transactions on Industry Applications, Vol. 24, No. 1, January/February 1988, pp. $142-150$.

2. Meynard, T.A. and Foch, H., "Dipositif électronique de conversion d'énergie électrique", French Patent No. 2679 715, Filed $25^{\text {th }}$ July 1991, Issued 29 $9^{\text {th }}$ January 1993.

3. Meynard, T.A. and Foch, H., "Multi-level choppers for high voltage applications", EPE Journal, Vol. 2, No. 1, March 1992, pp. 45 - 50.

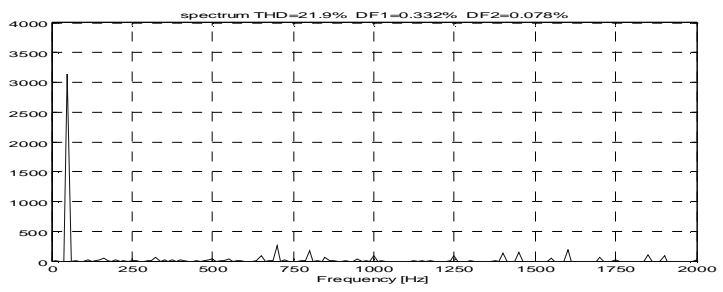

Figure 12: Method \#1 Line-to-Line Voltage Spectrum

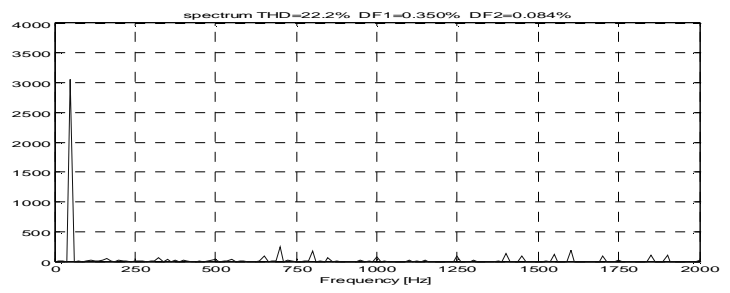

Figure 14: Method \#2 Line-to-Line Voltage Spectrum

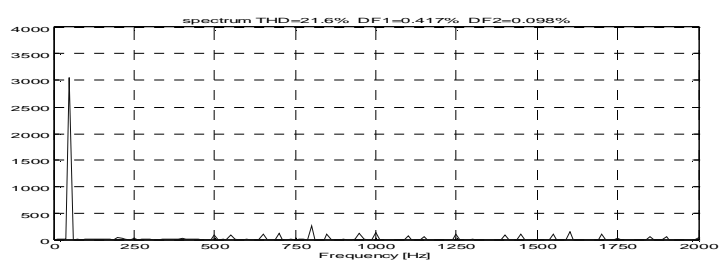

Figure 16: Method \#3 Line-to-Line Voltage Spectrum

4. Lee, Y.-H., Suk, B.-S. and Hyun, D.-S., "A novel PWM scheme for a three-level voltage source inverter with GTO thyristors", IEEE Transactions on Industry Applications, Vol. 32, No. 2, March/April 1996, pp. 260 - 268.

5. Zhang, H., Von Jouanne, A., Dai S., Wallace, A.K. and Wang, F., "Multilevel inverter modulation schemes to eliminate common-mode voltages", IEEE Transactions on Industry Applications, Vol, 36, No. 6 , Nov/Dec 2000, pp. $1645-1653$.

6. Peng, D., Lee, F.C. and Boroyevich, D., "A novel SVM algorithm for multilevel three-phase converters", Proceedings of PESC '02, June, 2002, Vol. 2, pp. 509 513.

7. Martins, C.A., Roboam, X., Meynard, T.A. and Carvalho, A.S., "Multi-level direct torque control with imposed switching frequency and reduced ripple", Proceedings of PESC '00, June, 2000, Vol. 1, pp. 435 441.

8. S.J. Watkins and L. Zhang, "Influence of multilevel sinusoidal PWM schemes on the performance of a flyingcapacitor inverter", Proceedings of PEMD '02, April 2002, pp. 92 - 97. 